\title{
Detecting quantitative trait loci for water use efficiency in rice using a recombinant inbred line population
}

\author{
ZHOU GuangSheng ${ }^{1}$, LIU Fang ${ }^{1}$, CAO JinHua ${ }^{1}$, YUE Bing $^{2} \&$ XIONG LiZhong ${ }^{2}$ \\ ${ }^{1}$ College of Plant Science and Technology, Huazhong Agricultural University, Wuhan 430070, China; \\ ${ }^{2}$ National Key Laboratory of Crop Genetic Improvement, Huazhong Agricultural University, Wuhan 430070, China
}

Received November 16, 2010; accepted January 21, 2011

\begin{abstract}
Breeding rice with high water use efficiency (WUE) can ameliorate water shortage through water-saving irrigation. However, WUE is a complex quantitative trait and very few studies have been conducted to measure WUE directly. In this study, a recombined inbred line population derived from a cross between an indica lowland rice and upland japonica rice was used to dissect the genetic control of WUE by fine-monitored water supply experiments. Quantitative trait loci (QTL) were scanned for 10 traits including heading date (HD), water-consumption per day (water/d), shoot weight gain per day (shootw/d), root weight gain per day (rootw/d), kernel weight gain per day (kernelw/d), average WUE at whole plant level (WUEwhole/d), average WUE for up-ground biomass (WUEup/d), average WUE for grain yield (WUEyield/d), average economic index (econindex/d), and average root/shoot ratio per day (ratio/d). The results show that most of the traits were significantly correlated to each other. Twenty-four QTL (LOD $\geqslant 2.0$ ) were detected for econindex, econindex/d, WUEyield, WUEyield/d, WUEup, WUEup/d, WUEwhole, WUEwhole/d, kernelw, kernelw/d, rootw, and water/d by composite interval mapping. These QTLs are located on chromosomes 1,2, 4, $6,7,8$, and 12. Individual QTLs accounted for 4.97\%-10.78\% of the phenotypic variation explained. Some of these QTLs overlapped with previously reported drought resistance QTLs detected in this population. These results provide useful information for further dissection of the genetic basis and marker-assisted selection of WUE in rice.
\end{abstract}

Oryza sativa, drought resistance, water use efficiency, quantitative trait loci, genetic mapping

Citation: Zhou G S, Liu F, Cao J H, et al. Detecting quantitative trait loci for water use efficiency in rice using a recombinant inbred line population. Chinese Sci Bull, 2011, 56: 1481-1487, doi: 10.1007/s11434-011-4444-9

Drought or water deficit is one of the major abiotic stresses limiting crop yield. Water shortage has become a global crisis and drought is also a serious threat to agriculture worldwide [1-3]. Besides the huge amount of fresh water consumed by agriculture, the water crisis will be increasingly severe considering the human population expansion and development of urbanization along with the fast-growing economies of many developing countries, which might further restrict the increase of food production [4]. Technologies for improving water use efficiency (WUE) of crops are therefore desperately required and it is also a major challenge to global agriculture [4-6]. Rice is the most important crop in Asian countries and shortage of water is the main factor restricting

*Corresponding author (email: lizhongx@mail.hzau.edu.cn) improvement in its yield. Rice consumes a lot of water with rather low WUE. Therefore, breeding rice with high WUE can ameliorate water shortage through water-saving irrigation, which has very important significance for food safety as well as environmental protection [7-9].

WUE is also an important criterion for evaluating yieldpotential of a given crop. Given the limited water resource, a crop with a higher WUE can produce superior biomass and/or economic yield [7]. The level of WUE varies in different crops such as rice, peanut, cowpea, cotton, sorghum, barley, wheat and soybean [4,7,10-12]. The WUE is commonly evaluated at 3 levels: single leaf (the ratio of net $\mathrm{CO}_{2}$ assimilation to transpiration of a single leaf) [7], plant [13] and population [4]. At the single leaf level, WUE can be also defined as the ratio of instantaneous carbon dioxide 
assimilation over transpiration [14], or as photosynthesis rate/transpiration rate that can be indirectly evaluated by discriminating the stable isotope of carbon $\left({ }^{13} \mathrm{C}\right)[7,15]$. In peanuts, WUE is positively related to specific leaf area determined by soil plant analysis development (SPAD) chlorophyll meter reading (SCMR) [16]. However, some researchers argue that these physiological indicators cannot directly reflect the WUE of leaves or breeding traits, especially that of economic output $[1,10,14,17]$. Previous studies suggest that improvement of leaf WUE may not necessarily result in higher crop WUE or yield [18], and carbon isotope measurement is expensive [16]. At the population level, WUE is usually defined as the ratio of the final crop yield over the total transpiration water in the field [14], and is frequently influenced by field management including fertilizer application, time of planting, soil water conditions, disease and pest control, and planting density. Nevertheless, water consumption is not easy to measure accurately, which is a big challenge for evaluating WUE in a large population and in breeding materials, in particular $[4,15]$. Direct measurement of WUE at the plant level has the merits of directly recording water consumption over the whole growth cycle and relevant indicators such as biomass (even including roots if necessary) and yield, and it is therefore considered the most accurate and trustworthy method for evaluating WUE of different varieties [7,8,10,19].

WUE is a complex quantitative trait. Yoichiro et al. [13] conducted quantitative trait locus (QTL) mapping of WUE using 106 backcross inbred lines (BILs) derived from a cross between Akihikari (lowland rice) and IRAT109 (upland rice) at the seedling stage ( $30 \mathrm{~d}$ from germination) under non-stressed and PEG 6000-stressed conditions. However, no QTL was identified under either condition. Xu et al. [8] detected seven QTLs for leaf WUE in a population of 98 BILs derived from a cross between temperate japonica and aus rice at the seedling stage (3-4 weeks from germination) with carbon isotope discrimination as the criterion. The QTL with the largest additive effect was from aus rice and was colocalized with QTLs for leaf length, tiller number, and nitrogen content [8]. Mian et al. [20] conducted QTL mapping on the WUE of beans with $120 \mathrm{~F}_{4}$ lines from the cross Young $\times$ PI416937 and detected four QTLs accounting for $38 \%$ of phenotypic variation.

This experiment was designed to investigate the genetic control of WUE at the plant/line level in rice using a recombined inbred line (RIL) population over the entire life cycle. Eleven QTLs were detected for five parameters related to WUE with $5.95 \%-10.78 \%$ of the phenotypic variation explained. The results in this study provide useful information for further dissection of the genetic basis of water use efficiency in rice.

\section{Materials and methods}

\subsection{Materials}

A RIL population derived from a cross between Zhenshan
97 (lowland indica rice) and IRAT109 (upland japonica rice) $[21,22]$ was preserved by our lab.

\subsection{Experimental design}

The parents and RIL population were evaluated for WUE. The experiments were laid out in a randomized complete-block design with four replications. For phenotyping, the plants were grown in plastic pots $(32 \mathrm{~cm}$ in diameter and $36 \mathrm{~cm}$ in depth) filled with $15 \mathrm{~kg}$ of sieved, sterilized sandy (paddy soileey sand=2:1), $0.5 \mathrm{~g}$ urea, and $4 \mathrm{~g} \mathrm{15-15-15}$ $(\mathrm{N}-\mathrm{P}-\mathrm{K})$ fertilizer. The experiment was conducted at the drought testing experimental station at Huazhong Agricultural University in 2007.

The RILs were direct-seeded in pots on June 5, 2007. In total, 728 pots ( 4 pots for each line of the 180 RILs and 2 parents) were placed under a rain-out shelter with movable roofs. Rain was excluded by closing the roof during periods of rain. At the 5-leaf stage, only one plant exhibiting vigorous growth was retained for each pot and the extra plants were removed. Each pot was then covered with a plastic film with a hole in the middle to allow normal plant growth, thus reducing the evaporation of water from the soil surface. Plants were irrigated to maintain a shallow water layer through the hole and the amount of water added was recorded every day during the entire life cycle.

\subsection{Trait evaluation}

All the plants were harvested when they were completely matured. The following traits were measured: heading date (HD), yield per plant, kernel weight gain per day (kernelw/d), total water consumption during the entire life cycle, average water consumption per day (water/d), dry root weight (rootw), root weight gain per day (rootw/d), dry shoot weight (shootw), shoot weight gain per day (shootw/d), dry weight of up-ground biomass, average root/shoot ratio per day (ratio/d), economic index, and average economic index per day $($ econindex/d). The samples were weighed after drying to a constant weight in a dry-case at $80^{\circ} \mathrm{C}$. The WUE was calculated with the following formulas:

Yield WUE (WUEyield) = kernel weight $(\mathrm{g}) /$ whole water consumption $(\mathrm{kg})$;

Up-ground biomass WUE (WUEup) = dry weight of up-ground biomass $(\mathrm{g}) /$ whole water consumption $(\mathrm{kg})$;

Whole WUE (WUEwhole) = dry weight of up-ground biomass and root $(\mathrm{g}) /$ whole water consumption $(\mathrm{kg})$.

With consideration of the variation in heading date among the RILs, each WUE trait value was divided by heading date for further analysis.

\subsection{Statistical analysis and QTL detection}

The distribution frequency, correlation coefficient, and variant analysis for each trait were analyzed with SAS 8.01 
(SAS Institute, Inc., Cary, North Carolina, USA). The heritability $\left(h^{2}\right)$ was calculated as: $h^{2}=\sigma_{\mathrm{g}}{ }^{2} /\left(\sigma_{\mathrm{g}}{ }^{2}+\sigma_{\mathrm{e}}{ }^{2} / r\right)$, where $\sigma_{\mathrm{g}}^{2}$ is the genetic variance, $\sigma_{\mathrm{e}}^{2}$ is the error variance and $r$ is the number of replications. The Shapiro-Wilk test was applied to test for normality among the traits.

Based on the linkage map constructed by Yue [21], composite interval QTL mapping was conducted to detect QTLs for WUE-related traits using WinQtl Cartographer 2.0 (statistical model 6) [23]. The threshold of $2.0(\mathrm{LOD} \geqslant 2.0)$ was chosen for claiming putative QTLs. The additive effect, dominance effect and phenotypic variation explained by individual QTLs were also obtained according to the software analysis.

\section{Results}

\subsection{Analysis of phenotypic data}

The average values of all the traits for the two parents and RILs are listed in Table 1. The average trait values of Zhenshan97 $\left(\mathrm{P}_{1}\right)$ are larger than those of IRAT109 $\left(\mathrm{P}_{2}\right)$ for all the traits except heading date, water/d and shootw/d, which suggests that, in general, Zhenshan97 may have a higher WUE than IRAT109. The difference in rootw/d and ratio/d between the 2 parents was significant. The RILs exhibited a wide range of variation for the traits investigated. All the traits showed transgressive and continuous distributions, suggesting polygenic inheritance of the traits. Transgressive segregation in both directions was observed for most traits, indicating that both parents may have favorable alleles for the traits.

As shown in Table 1, the highest heritability $\left(h^{2}\right)$ of the traits was 0.97 for WUEwhole/d and WUEup/d. The other traits also displayed high heritability except for econindex/d and ratio/d, which indicates that the WUE-related traits in rice have rather high heritability and might have a significant effect when used for selection in breeding rice with high WUE. Compared with WUEwhole/d and WUEup/d, econindex/d and ratio/d had rather low heritability.

Correlation analysis was performed for all of the measured traits in the RIL population (Table 2). The results show that strong correlations existed between all per-day traits (i.e. trait value/d) and heading date (HD), suggesting that the per-day traits are suitable to evaluate WUE. The majority of the other traits showed significant correlation to each other, among which WUEup/d and WUEwhole/d showed the highest correlation. The WUEyield/d was highly $(r=$ 0.94) correlated to econindex/d.

Table 1 Summary statistics of all water use efficiency (WUE)-related traits for both parents (Zhenshan97 and IRAT109) and the recombined inbred line (RIL) population

\begin{tabular}{|c|c|c|c|c|c|c|c|c|}
\hline \multirow{2}{*}{$\begin{array}{c}\text { Trait } \\
\text { Heading date (HD) }\end{array}$} & \multirow{2}{*}{$\begin{array}{c}\text { Zhenshan97 }\left(\mathrm{P}_{1}\right) \\
94\end{array}$} & \multirow{2}{*}{$\frac{\text { IRAT109 }\left(\mathrm{P}_{2}\right)}{100}$} & \multirow{2}{*}{$\frac{\mathrm{P}_{1} \mathrm{vs} \cdot \mathrm{P}_{2}^{\mathrm{a})}}{\mathrm{ns}}$} & \multicolumn{2}{|c|}{ RIL (mean) $)^{\text {b) }}$} & \multicolumn{2}{|c|}{ RIL (range) } & \multirow{2}{*}{$\begin{array}{l}h^{2} \\
-\end{array}$} \\
\hline & & & & 101.45 & \pm 11.05 & 83 & -132 & \\
\hline Water/d $\left(\mathrm{mL} \mathrm{d}^{-1}\right)$ & 262.84 & 266.33 & ns & 251.48 & \pm 40.97 & 87.48 & -355.51 & 0.87 \\
\hline Shootw/d $\left(\mathrm{g} \mathrm{d}^{-1}\right)$ & 0.39 & 0.42 & ns & 0.50 & \pm 0.12 & 0.15 & -0.96 & 0.8 \\
\hline Rootw/d $\left(\mathrm{g} \mathrm{d}^{-1}\right)$ & 0.11 & 0.08 & $* *$ & 0.10 & \pm 0.04 & 0.01 & -0.21 & 0.79 \\
\hline Kernelw/d $\left(\mathrm{g} \mathrm{d}^{-1}\right)$ & 0.36 & 0.35 & $\mathrm{~ns}$ & 0.27 & \pm 0.08 & 0.09 & -0.44 & 0.74 \\
\hline WUEwhole/d $\left(10^{-3} \mathrm{~g} \mathrm{~kg}^{-1} \mathrm{~d}^{-1}\right)$ & 35.35 & 31.63 & ns & 34.18 & \pm 3.88 & 24.08 & -48.33 & 0.97 \\
\hline WUEup/d $\left(10^{-3} \mathrm{~g} \mathrm{~kg}^{-1} \mathrm{~d}^{-1}\right)$ & 30.67 & 28.6 & ns & 30.43 & \pm 3.69 & 21.29 & -43.69 & 0.97 \\
\hline WUEyield/d $\left(10^{-3} \mathrm{~g} \mathrm{~kg}^{-1} \mathrm{~d}^{-1}\right)$ & 14.79 & 12.97 & ns & 10.84 & \pm 3.24 & 3.75 & -17.53 & 0.88 \\
\hline Econindex/d $\left(10^{-3} \mathrm{~d}^{-1}\right)$ & 5.13 & 4.54 & ns & 3.59 & \pm 1.11 & 1.16 & -5.93 & 0.24 \\
\hline Ratio/d $\left(10^{-3} \mathrm{~d}^{-1}\right)$ & 1.61 & 1.06 & $* *$ & 1.33 & \pm 0.41 & 0.38 & -4.55 & 0.27 \\
\hline
\end{tabular}

a) Statistical test for difference between the two parents at $P<0.05(*)$ and $P<0.01(* *)$ levels. ns, not significant; b) Data represent mean and standard error.

Table 2 Correlation analysis between the water use efficiency (WUE)-related traits in the RIL population ${ }^{\text {a) }}$

\begin{tabular}{|c|c|c|c|c|c|c|c|c|c|}
\hline Trait & HD & Water/d & Shootw/d & Rootw/d & Kernelw/d & WUEwhole/d & WUEup/d & WUEyield/d & Econindex/d \\
\hline Water/d & $-0.27^{* *}$ & & & & & & & & \\
\hline Shootw/d & $0.30^{* * *}$ & $0.54^{\text {*** }}$ & & & & & & & \\
\hline Rootw/d & $0.46^{* *}$ & $0.46^{* *}$ & $0.76^{* * *}$ & & & & & & \\
\hline Kernelw/d & $-0.51^{\text {*** }}$ & $0.61^{\text {*** }}$ & -0.08 & -0.01 & & & & & \\
\hline WUEwhole/d & $-0.49^{* * *}$ & 0.09 & $0.26^{* *}$ & 0.1 & $0.35^{* * *}$ & & & & \\
\hline WUEup/d & $-0.60^{\text {*** }}$ & 0.04 & 0.13 & -0.12 & $0.40^{\text {** }}$ & $0.97^{* *}$ & & & \\
\hline WUEyield/d & $-0.69^{* *}$ & 0.13 & $-0.48^{* *}$ & $-0.40^{* *}$ & $0.82^{* * *}$ & $0.47^{* *}$ & $0.57^{* *}$ & & \\
\hline Econindex/d & $-0.76^{* *}$ & $0.19^{*}$ & $-0.59^{* * *}$ & $-0.48^{* *}$ & $0.78^{* * *}$ & $0.24^{\text {** }}$ & $0.36^{* *}$ & $0.94^{* * *}$ & \\
\hline Ratio/d & $0.35^{* *}$ & $0.21^{* * *}$ & $0.42^{* *}$ & $0.72^{* *}$ & $-0.16^{*}$ & -0.04 & $-0.21^{* *}$ & $-0.36^{* *}$ & $-0.36^{* * *}$ \\
\hline
\end{tabular}

a) * and ** mean significance level at $P \leqslant 0.05$ and $P \leqslant 0.01$, respectively. 


\subsection{QTL analysis of WUE-related traits}

The entire genome was scanned for QTLs conferring a significant effect on the WUE-related traits by composite interval mapping in the RIL population. The putative QTLs detected are presented in Table 3. A graphical presentation of QTL locations on the linkage map is shown in Figure 1. Twenty-four QTLs were detected with 12 traits involved. Individual QTLs accounted for $4.97 \%-10.78 \%$ of the phenotypic variation explained. The LOD values of these 24 QTLs ranged from 2.0 to 4.4 , while their additive effect ranged from -16.282 to 11.362 , and their dominance effect ranged from 0.0552 to 19.4486 .

One QTL for econindex was detected on Chr1 (RM5RM237), which explained $5.12 \%$ of phenotypic variation. The allele for increasing trait value was from Zhenshan97.

One QTL located on Chr4 and 3 adjacent QTLs located on Chr6 were identified for econindex/d. The QTL located on Chr4 accounted for $8.20 \%$ of the trait variation, and the 3 QTLs located on Chr6 accounted for $10.78 \%, 8.94 \%$ and $8.71 \%$ of the trait variation, respectively. All the alleles of these QTLs from IRAT109 had a positive effect on increasing trait value.

Two QTLs located on Chr4 (RM119-RM273) and Chr2
(RM324-RM301) were detected for WUEyield and accounted for $5.75 \%$ and $5.99 \%$ of the trait variation, respectively.

Two QTLs controlling WUEup were identified on Chr1 (RM5-RM237) and Chr2 (RM573-RM318) and accounted for $6.79 \%$ and $5.42 \%$ of the phenotypic variation, respectively. One QTL of WUEup/d was detected in the same region of the QTL for WUEup (RM573-RM318) on Chr2, explaining $7.33 \%$ of phenotypic variation. All alleles of these QTLs for increasing trait values were from Zhenshan97.

Two QTLs were detected for WUEwhole. They were located on Chr1 (RM5-RM237) and Chr2 (RM573-RM318) and explained $7.18 \%$ and $8.97 \%$ of the trait variation, respectively. The alleles for increasing trait values were both from Zhenshan97.

Three QTLs controlling kernelw were identified on Chr4 (RM119-RM273), Chr7 (RM418-RM432) and Chr8 (RM350-RM284). The first and the third QTL had higher LOD values (3.5 and 3.9) and accounted for higher phenotypic variation $(8.46 \%$ and $9.20 \%)$ than the second QTL.

Five QTLs were identified for kernelw/d, in total accounting for $39.33 \%$ of the phenotypic variation. Three of them overlapped with the regions of the 3 kernelw QTLs, while the other 2 QTLs were tandem located on Chr6.

Table 3 Map position and main characteristics of QTLs with a LOD score $\geqslant 2.0$ for WUE-related traits

\begin{tabular}{|c|c|c|c|c|c|c|c|c|}
\hline \multirow{2}{*}{$\begin{array}{c}\text { Trait } \\
\text { Econindex }\end{array}$} & \multirow{2}{*}{$\begin{array}{c}\text { Chr } \\
1\end{array}$} & \multirow{2}{*}{$\begin{array}{c}\text { Position (cM) } \\
144.07\end{array}$} & \multicolumn{2}{|c|}{ Interval } & \multirow{2}{*}{$\frac{\text { LOD }}{2.1}$} & \multirow{2}{*}{$\begin{array}{l}\text { Additive }^{\text {a) }} \\
-0.0577\end{array}$} & \multirow{2}{*}{$\begin{array}{c}\text { Dominance } \\
2.3167\end{array}$} & \multirow{2}{*}{$\begin{array}{c}\operatorname{Var}^{\text {b) }} \\
5.12\end{array}$} \\
\hline & & & RM5 & -RM237 & & & & \\
\hline \multirow{4}{*}{ Econindex/d } & 4 & 38.91 & RM4142 & $-\mathrm{RM} 119$ & 2.3 & 0.0003 & 0.4409 & 8.20 \\
\hline & 6 & 106.36 & RM454 & -MRG4371 & 2.8 & 0.0004 & 1.0137 & 10.78 \\
\hline & 6 & 117.58 & MRG4371 & -RM528 & 2.8 & 0.0003 & 0.8064 & 8.94 \\
\hline & 6 & 123.1 & RM528 & $-\mathrm{RM} 30$ & 2.7 & 0.0003 & 0.7225 & 8.71 \\
\hline WUEyield & 4 & 45.04 & RM119 & $-\mathrm{RM} 273$ & 2.2 & 0.0667 & 0.5828 & 5.75 \\
\hline WUEyield/d & 2 & 71.67 & RM324 & -RM301 & 2.3 & -0.0011 & 1.8709 & 5.99 \\
\hline \multirow{2}{*}{ WUEup } & 1 & 146.07 & RM5 & -RM237 & 2.3 & -0.0824 & 0.1725 & 6.79 \\
\hline & 2 & 153.58 & RM573 & -RM318 & 2.3 & -0.0745 & 0.0738 & 5.42 \\
\hline WUEup/d & 2 & 153.58 & RM573 & -RM318 & 3.0 & -0.0010 & 0.0552 & 7.33 \\
\hline WUEwhole & 1 & 146.07 & RM5 & -RM237 & 2.4 & -0.1000 & 1.9874 & 7.18 \\
\hline WUEwhole/d & 2 & 153.58 & RM573 & -RM318 & 3.5 & -0.0012 & 0.9638 & 8.97 \\
\hline \multirow[t]{4}{*}{ Kernelw } & 7 & 80.26 & RM418 & $-\mathrm{RM} 432$ & 2.2 & 1.7424 & 1.8904 & 4.97 \\
\hline & 8 & 74.95 & RM350 & -RM284 & 3.9 & -2.5886 & 1.8931 & 9.20 \\
\hline & 4 & 47.22 & RM119 & $-\mathrm{RM} 273$ & 3.4 & 0.0253 & 1.2607 & 9.08 \\
\hline & 6 & 108.36 & RM454 & -MRG4371 & 2.8 & 0.0265 & 1.7921 & 9.12 \\
\hline \multirow[t]{3}{*}{ Kernelw/d } & 6 & 117.58 & MRG4371 & -RM528 & 3.0 & 0.0253 & 0.9281 & 8.48 \\
\hline & 7 & 82.26 & RM418 & $-\mathrm{RM} 432$ & 2.7 & 0.0225 & 1.2274 & 6.70 \\
\hline & 8 & 74.95 & RM350 & -RM284 & 2.7 & -0.0210 & 1.1633 & 5.95 \\
\hline \multirow[t]{2}{*}{ Rootw } & 12 & 87.10 & MRG4479 & $-\mathrm{RM} 235$ & 2.4 & 1.9177 & 10.2959 & 5.58 \\
\hline & 7 & 85.07 & RM432 & -RM182 & 2.8 & 11.3620 & 19.4486 & 7.35 \\
\hline \multirow[t]{2}{*}{ Water/d } & 8 & 53.07 & RM404 & -RM339 & 2.0 & 10.6510 & 18.4231 & 5.03 \\
\hline & 8 & 72.11 & RM350 & -RM284 & 4.4 & -16.2816 & 18.3111 & 10.54 \\
\hline
\end{tabular}

a) Additive effects, the positive values indicate alleles from IRAT109 have the effect of increasing the trait value. b) Phenotypic variation explained by the QTL. 

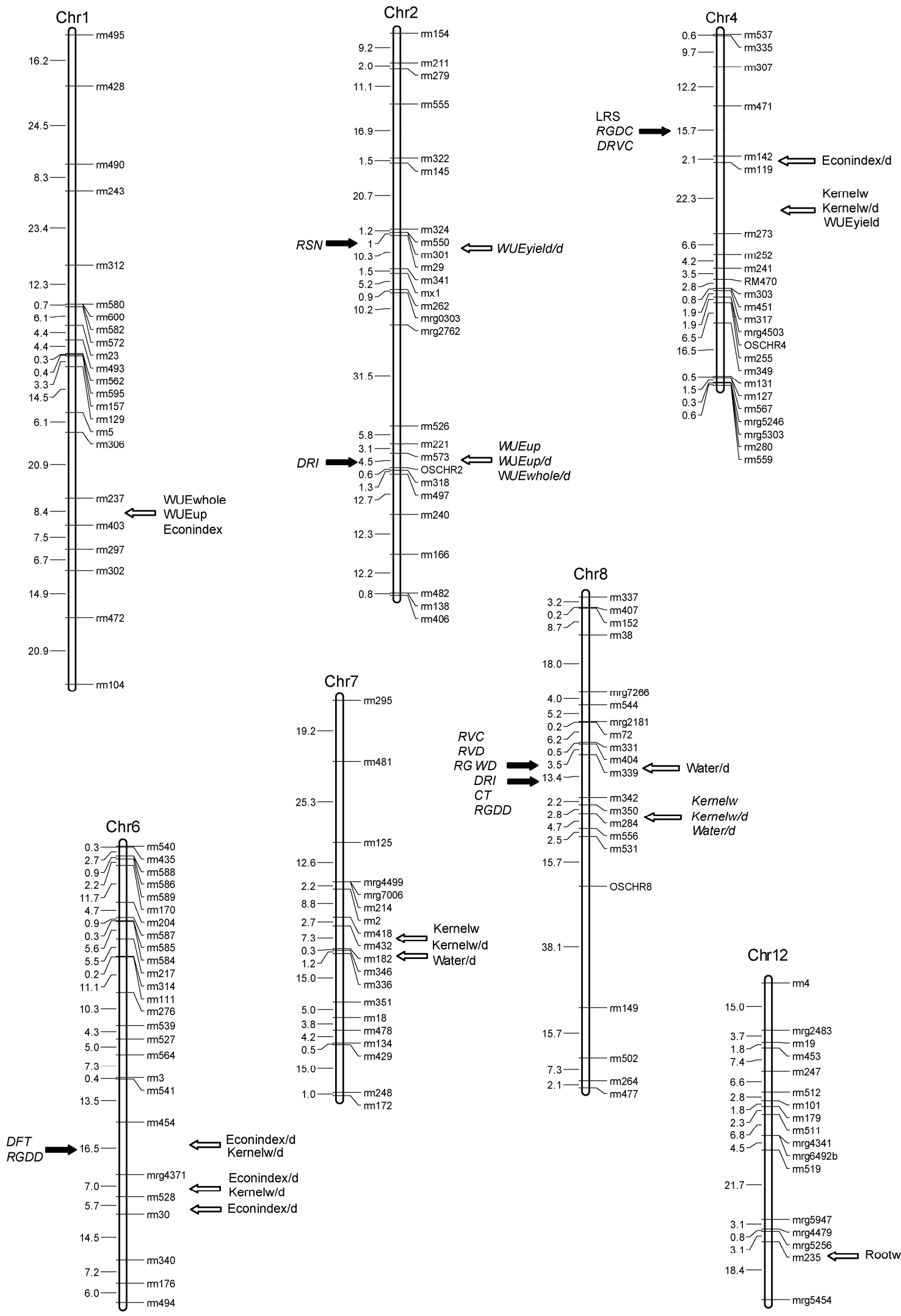

Figure 1 Quantitative trait loci (QTLs) detected in the recombinant inbred line population. The QTLs detected in this study are shown on the right of the chromosomes, and the drought resistance-related QTLs detected by Yue [21] and Yue et al. [21,22] are marked on the left. Italicized QTLs indicate that the alleles for increasing trait values were from Zhenshan 97. 
Except for the QTL on Chr8, all alleles positively contributing to the trait value were from IRAT109.

One QTL was detected for rootw on Chr12 (MRG4479RM235), explaining 5.58\% of phenotypic variation with the allele for increasing trait value from IRAT109.

Three QTLs controlling water/d were detected on Chr7 (RM432-RM182), Chr8 (RM404-RM339), and Chr8 (RM350-RM284). The QTL on Chr8 (RM350-RM284) had the highest LOD value (4.4) and accounted for a higher proportion of phenotypic variation $(10.51 \%)$.

We noted a number of obvious QTL clusters in this study. QTLs controlling WUEwhole, WUEup and econindex were identified in the region RM237-RM403 on Chr1. QTLs controlling WUEup, WUEup/d and WUEwhole/d were identified in the region RM573-RM318 on Chr2. QTLs controlling kernelw, kernelw/d and WUEyield were identified in the region RM119-RM273 on Chr4. QTLs controlling econindex/d and kernelw/d were both identified in the region M454-MRG4371 on Chr6. QTLs controlling econindex/d and kernelw/d, both identified in the region MRG4371-RM528, were also located in adjacent regions on Chr6. QTLs detected in the region RM350-RM284 of Chr8 controlled kernelw, kernelw/d and water/d. Distribution characteristics displayed by these QTL were in accordance with correlations among these traits (Table 2).

\section{Discussion}

In this study, QTL mapping of rice WUE-related traits was conducted using a RIL population. To accurately monitor the water consumption, the water supply of each plant of the parents and RILs were recorded daily and the pots were covered with thin plastic film to reduce variation in the evaporation of water from the soil surface. Actual water consumption per plant per day is recorded and the average or per-day WUE was calculated. Therefore, the results from this study directly and accurately reflect WUE for the traits investigated, although the entire process is time-consuming.

Greater WUE represents a strategy by which plants can increase the production of biomass when growing in water-limiting conditions [10]. However, crop WUE is a complex characterisitc. The WUE at leaf level, net photosynthetic rate $(\mathrm{Pn})$, and leaf transpiration efficiency are closely connected. The WUE at biomass and yield level is not only closely related to genetic variation in water uptake, but also to genetic variation in photoassimilation and distribution of photosynthesis products [24]. In this experiment, a QTL for WUEyield/d was detected in the region (RM324-RM301) on $\mathrm{Chr} 2$, the same region in which the QTL controlling RSN (relative spikelets number per panicle) was detected [21]; QTLs for WUEup/d and WUEwhole/d were detected in the region (RM573-RM318) on $\mathrm{Chr} 2$, the same region as the QTL controlling DRI (drought d traits; two QTLs for econindex/d and another two QTLs for kernelw/d are posi- tioned in 2 neighboring intervals (RM454-MRG4371 and MRG4371-RM528) on Chr6, among which the former is the same QTL interval for DFT (delayed flowering time under drought stress) and the root trait RGDD (root growth under drought stress) detected by Yue [21], suggesting that genes with multiple function might also exist in this region.

Two QTLs each controlling kernelw/d and water/d, respectively, were detected in the interval RM350-RM284 on Chr8; the QTL for water/d had the highest LOD among all QTLs detected in this study. This region is adjacent to RM339-RM342, where DRI and CT (canopy temperature)-related QTLs [20] and RGDD-related QTL are positioned [21]. One QTL controlling kernelw/d was located on Chr4 and this region neighbors the region (RM142-RM119) where one QTL controlling kernelw is positioned. Two QTLs on Chr7 each controlling kernelw/d (RM418RM432) and water/d (RM432-RM182), respectively, were adjacent to each other. However, no drought resistancerelated QTLs were detected in these regions [21].

Drought resistance mechanisms of crops are rather complicated and most drought-adaptive traits are under polygenic control [24], which is the main reason that effective and feasible drought evaluation methods are as yet unavailable for breeding practice [17]. WUE is an important trait associated with drought resistance of crop plants [19], and crop yield under drought is related to water uptake, WUE, and harvest index. Genetic improvement of WUE could enhance the drought resistance of crops [17]. Mian et al. [19] reported that WUE is one of the physiological traits associated with drought tolerance in soybean. Hufstetler et al [11]. noted that WUE was significantly and negatively correlated with leaf epidermal conductance during vegetative growth in a study of 12 bean varieties. Impa et al. proposed that WUE has the most intimate relationship with drought tolerance in rice. In this study, the positions of most QTLs related to WUE overlap with QTLs related to drought resistance. These results suggest that WUE and drought resistance could be simultaneously improved by selecting the overlapping QTLs.

In conclusion, this study conducted initial mapping of QTLs for traits related to WUE of rice. The results provide a useful reference for further dissecting the genetic and physiological basis of WUE in rice.

This work was supported by the National Natural Science Foundation of China (30725021 and 30921091).

1 Abdelbagi M I, Anthony E H. Inheritance of carbon isotope discrimination and water-use efficiency in cowpea. Crop Sci, 1993, 33: 498-503

2 Akihiko K, Babu R C, Boopathib N M, et al. Phenotypic and genotypic analysis of drought-resistance traits for development of rice cultivars adapted to rainfed environments. Field Crops Res, 2008, 109: $1-23$

3 Boyer J S. Plant productivity and environment. Science, 1982, 218: 443-448

4 Howell T A. Enhancing water use efficiency in irrigated agriculture. 
Agron J, 2001, 93: 281-289

5 Krishnamurthy L, Vadez V, Jyotsna D M, et al. Variation in transpiration efficiency and its related traits in a groundnut (Arachis hypogaea L.) mapping population. Field Crops Res, 2007, 103: 189-197

6 Zhang Z B, Shao H B, Xu P, et al. On evolution and perspectives of bio-watersaving. Colloids Surf B Biointerfaces, 2007, 55: 1-9

7 Impa S M, Nadaradjan S, Boominathan P, et al. Carbon isotope discrimination accurately reflects variability in WUE measured at a whole plant level in rice. Crop Sci, 2005, 45: 2517-2522

8 Xu Y B, Dominique T, Roman C P, et al. Leaf-level water use efficiency determined by carbon isotope discrimination in rice seedlings: Genetic variation associated with population structure and QTL mapping. Theor Appl Genet, 2009, 118: 1065-1081

9 Zhao X Q, Xu J L, Zhao M, et al. QTLs affecting morph-physiological traits related to drought tolerance detected in overlapping introgression lines of rice (Oryza sativa L.). Plant Sci, 2008, 174: 618-625

10 Hugh J E. Stomatal and non-stomatal restrictions to carbon assimilation in soybean (Glycine max) lines differing in water use efficiency. Environ Exp Bot, 2002, 48: 237-246

11 Hufstetler E V, Boerma H R, Carter T E, et al. Genotypic variation for three physiological traits affecting drought tolerance in soybean. Crop Sci, 2007, 47: 25-35

12 Zhang X Y, Chen S Y, Liu M Y, et al. Improved water use efficiency associated with cultivars and agronomic management in the north China plain. Agron J, 2005, 97: 783-790

13 Yoichiro K, Sawa H, Keisuke N, et al. Identification of QTLs controlling rice drought tolerance at seedling stage in hydroponic culture. Euphytica, 2008, 160: 423-430
14 Mikhail A S, Pierre M, Peter D J. Quantifying effects of simple wheat traits on yield in water-limited environments using a modeling approach. Agricult Forest Meteorol, 2009, 149: 1095-1104

15 Bjorn M, Charles G T, Robert K L. Carbon isotope discrimination as a tool to improve water-use efficiency in tomato. Crop Sci, 1999, 39: 1775-1783

16 Upadhyaya H D. Variability for drought resistance related traits in the mini core collection of peanut. Crop Sci, 2005, 45: 1432-1440

17 Specht J E, Chase K, Macrander M, et al. Soybean response to water: A QTL analysis of drought tolerance. Crop Sci, 2001, 41: 493-509

18 Condon A G, Richards R A, Rebetzke G J, et al. Breeding for high water-use efficiency. J Exp Bot, 2004, 55: 2447-2460

19 Mian M A, Ashley D A, Boerma H R. An additional QTL for water use efficiency in soybean. Crop Sci, 1998, 38: 390-393

20 Mian M A R, Bailey M A, Ashley D A, et al. Molecular markers associated with water use efficiency and leaf ash in soybean. Crop Sci, 1996, 36: 1252-1257

21 Yue B. Study on the genetic basis of drought resistance at reproductive stage in rice. Doctor Dissertation. Wuhan: Huazhong Agricultural University, 2005

22 Yue B, Xiong L Z, Xue W Y, et al. Genetic analysis for drought resistance of rice at reproductive stage in field with different types of soil. Theor Appl Genet, 2005, 111: 1127-1136

23 Zeng Z B. Precision mapping of quantitative trait loci. Genetics, 1994, 136: 1457-1468

24 Zhao H, Zhang Z B, Shao H B, et al. Genetic correlation and path analysis of transpiration efficiency for wheat flag leaves. Environ Exp Bot, 2008, 64: 128-134

Open Access This article is distributed under the terms of the Creative Commons Attribution License which permits any use, distribution, and reproduction in any medium, provided the original author(s) and source are credited. 